\title{
Evaluation Criteria for True (Physical) Random Number Generators Used in Cryptographic Applications
}

\author{
Werner Schindler ${ }^{1}$ and Wolfgang Killmann ${ }^{2}$ \\ 1 Bundesamt für Sicherheit in der Informationstechnik (BSI) \\ Godesberger Allee 185-189 \\ 53175 Bonn, Germany \\ Werner.Schindler@bsi.bund.de \\ 2 T-Systems ISS GmbH \\ Rabinstr. 8 \\ 53111 Bonn, Germany \\ Wolfgang.Killmann@t-systems.com
}

\begin{abstract}
Random number generators are essential components of many cryptographic systems. Inappropriate random number generators may weaken the security properties of the system considerably. This paper considers evaluation criteria for true (physical) random number generators. General objectives are formulated and possible criteria and measures are discussed which shall ensure these goals. Central parts of the mathematical-technical reference of the German evaluation guidance document AIS $31([19 \mid 2])$ are cited and rationale is given.
\end{abstract}

Keywords: True random number generator, entropy, evaluation criteria, online test.

\section{Introduction}

Many security mechanisms need nonrecurring and / or unpredictable data as nonces or secrets. While the non-recurrence property is fulfilled for nearly all random number generators (RNGs) with overwhelming probability (provided that the length of the (pseudo-)random strings is sufficiently large) unpredictability is more difficult to assure. An RNG with this property which can neither be observed nor controlled by an attacker generates ideal secrets like cryptographic keys. Random numbers are needed by many cryptographic applications. They are used for the generation of random session keys, signature keys and signature parameters, challenges and zero knowledge proofs, for instance.

Ideal random number generators are characterized by the property that the generated random numbers are independent and uniformly distributed on a finite range. An ideal random number generator, however, is a fiction. The class of (real world) RNGs falls into three subclasses. First, random microcosmic processes may cause physical effects applicable as random noise sources. Examples 
are quantum random processes, time between emissions during radioactive decay, inherent semiconductor thermal noise, shot noise from Zener diodes or freerunning oscillators. These processes are chaotic and non-deterministic by their nature. Normally, the random noise source produces a time-continuous analog signal which is digitised after uniform time intervals (e.g. by a comparator) which yield the so-called digitized analog signals, briefly denoted as das random numbers. Such RNGs are called true or physical (TRNG) because of their random noise source. In many designs the das random numbers are algorithmically postprocessed in order to reduce or at least to mask potential weaknesses. This gives the so-called internal random numbers. Upon external call the TRNG outputs external random numbers. TRNG are implemented e.g. in smart cards.

Pseudorandom number generators (or synonymously: deterministic random number generators, DRNGs) form the second subclass. They generate pseudorandom numbers deterministically from a randomly chosen seed. The third subclass are the 'hybrid generators' which refresh their seed regularly, for instance by means of random numbers derived from user's interaction (mouse movement or key strokes) and / or register values of the used PC. Applying an appropriate transformation (usually a compression algorithm) yields the desired random numbers.

In the past a lot of research work has been devoted to the development of good physical noise sources (see [42114], for instance), and a variety of deterministic and hybrid random number generators have been proposed. Less work has been spent in the development of suitable tests and assessment criteria.

Random numbers are not only needed for cryptographic applications but also for Monte Carlo methods and, above all, in stochastic simulations. Stochastic simulations treat probabilistic problems which cannot be solved analytically because they are too complex (consider the service times in complex multiuser systems, for example). Roughly speaking, one generates pseudorandom numbers ([1220]) with statistical properties depending on the specific simulation problem. Repeating this process many times one hopes to get reliable estimators for the unknown solution of the underlying probabilistic problem (typically a distribution of a random variable or a random vector or any restriction of it). A large number of statistical tests and whole test suites have been proposed to assess the statistical properties of the generated pseudorandom numbers ([20,22]) as unsuitable pseudorandom number generators may suggest false conclusions. These test suites are also often applied to random numbers used for sensitive cryptographic applications. However, besides statistical properties sensitive cryptographic applications demand that the used random numbers should have a backward and forward unpredictability property (cf. Sect. 2). As will be explained below this property cannot be assured by applying statistical blackbox tests. However, also [24 25 27] provide blackbox test suites. (Note that in [27] support is given how to apply the statistical tests, and in Sect. 1.1.2 it is noted explicitly that random numbers should be unpredictable. However, similarly as in [13], for instance, clear criteria are missing which assure this goal.) In [19] an approach for the evaluation of physical random number generators (due to CC (Common Crite- 
ria; cf. 8]) or ITSEC (Information Technology Security Evaluation Criteria; cf. [15])) is given which takes the construction of the TRNG explicitly into account. [19] is the mathematical-technical reference of the AIS 31 (2]) which has been effective in the German evaluation and certification scheme since September 2001. In the present paper we will explain the main items of [19] and the central ideas behind them. We point out that there is a similar document for deterministic random number generators ([129]; cf. Section 2 for a brief discussion).

In Sect. 2 the fundamental differences between true, deterministic and hybrid generators are explained. In Sect. 3 the general objectives of a TRNG evaluation are briefly pointed out whereas Sects. 4, 5and 6 go into detail. Generic requirements are formulated and concrete measures and criteria from [19] are given which shall ensure these requirements. The paper ends with considerations concerning the vulnerablity analysis and final remarks.

\section{True, Deterministic, and Hybrid Random Number Generators: Main Differences}

RNGs generally consist of a random source and a deterministic postprocessing. Of course, if a TRNG has an appropriate noise source a deterministic postprocessing after the digitization of the analog signal is not necessary. Design and analysis of an RNG is based on the understanding of randomness.

We already mentioned physical processes as random sources. Another kind of randomness is based of the intersection of causal chains: the combination of events of several processes which are independent of each other may behave randomly. Consider, for example, system data as time of interrupts, hard-disk seek times or user interactions. The randomness of the combined random source will increase with the number of the processes, their 'degree' of independence and the randomness within each of the processes. These processes are part of the RNG and cannot be neglected in its analysis even if the RNG is mostly implemented in software. An example of such a software RNG can be found in OpenSSL implementations under Windows operating systems.

The postprocessing may transform the digitized random signals into uniformly distributed random numbers even if the initial signal has significant statistical defects. Moreover, the postprocessing may collect entropy of the random noise source but it does not stretch the digitized random signal into longer strings.

Deterministic random number generators rely on a completely different concept. Viewed as an autonomous automaton a DRNG has an internal state whose initial value is called the seed, a state transition function and a output function. Such automatons are very cheap to implement as they merely require some additional lines of code. Their drawback lies in the fact that the seed (generated by a true or hybrid generator) contains the overall entropy of all pseudorandom numbers which are generated until a new seed is chosen. Therefore the evaluation methodology [29], for instance, requires a clear description of how the seed is generated together with a rationale why this will induce a specified distribution. 
Depending on the applications the DRNG is designated for this methodology distinguishes four DRNG classes K1 to K4. Class K1 generators only have to produce mutually different vectors with high probability whereas their statistical properties are irrelevant. Such vectors might be used for challenge-response protocols, for instance. The higher classes extend the possible applications of the random numbers to the generation of key or signature parameters. The requirements increase from mutually different outputs, through statistical features of the output (class K2), minimum bounds for the entropy of the seed and the practical impossibility for an adversary to work out preceding and following random numbers of a known part of the output sequence (class K3). Class K4 additionally demands that an adversary shall not be able to determine preceding random numbers even if he knows the actual internal state of the DRNG. An example of a K4-DRNG is the NIST approved DRNG for the DSA (see [26], Annex 3 and Change Notice 1) without the optional user input (provided that the seed is generated in an appropriate manner).

While the overall entropy of the output sequence of a true RNG increases with each random number, the entropy of the output of a DRNG is limited by the entropy of the seed initializing the internal state. Hybrid RNGs try to compensate the drawback of DRNGs by regular re-seeding the internal state by a random source (like user's interaction, register values of a PC or, more favourably, by a TRNG) and stretching the internal state like a DRNG. We note in this context that the randomness induced by the interaction of a user or by register values of a PC is difficult to assess. For example LINUX implements a hybrid RNG with a combined random source as /dev/urandom. The ANSI $\mathrm{X} 9.17$ RNG 3] refreshes the seed by means of the time which has only little entropy and might be guessed. Interestingly, the RNG [26], Annex 3, may be weak in hybrid mode, namely if an attacker is able to control the optional user input (see [18] for details).

It is important to note that a finite set of statistical tests may detect defects of a random source. On the other hand these tests cannot verify the randomness of the source. Moreover, the needs of IT security and especially of cryptography are different from those of stochastic simulations.

Remark 1. The Kolmogorov complexity theory defines a sequence as random if the length of the shortest program that generates a segment of the sequence arises with the length of this segment. A cryptographic strong definition of randomness requires pseudo-random sequences to be computationally indistinguishable from a randomly chosen uniformly distributed sequence (cf. [7]).

In the context of cryptography we may be faced with an active attacker who does not only analyze the RNG design and its output but additionally tries to affect or control the RNG. We hence should not only have a theoretical abstraction of an RNG in mind but also its concrete realization. 


\section{General Objectives on a TRNG Evaluation}

Normally, random number generators are part of an IT security product whose overall security (or particular aspects thereof) has to be assessed. The random number generation may be an externally visible security function, e.g. provided by a smart card chip to the operating system for the key generation. However, requirements on the TRNG often depend on the internal function they provide for other cryptographic components.

Roughly speaking, a TRNG evaluation falls into two phases. At first, the suitability of the random number generation process has to be checked at hand of some prototypes. In Sects. 4 and 5 this phase is considered in detail.

However, even if the design of the TRNG has turned out to be convenient this does not necessarily imply that each TRNG of the same production run has the same pleasant properties as the prototypes in the lab all the time when it is in operation. Tolerances of components of the random noise source maybe responsible that the 'quality' of the actually produced random numbers is worse than that of the carefully investigated prototypes. Also aging of the components or even the impact of an attacker may affect the characteristics of the generated das random numbers. In a worst case scenario the noise source may totally break down, maybe for natural reasons or as a consequence of a successful attack. The das random numbers are constant from then on, and the total entropy of the das random number sequence does not increase any more.

In order to detect such defects TRNGs should perform start-up tests, online test and so-called tot tests while they are in operation (cf. Sect. 6). The evaluation should give evidence that these measures are appropriate to assure this goal.

ITSEC (Information Technology Security Evaluation Criteria) and CC (Common Criteria; cf. 8]) provide evaluation criteria. ITSEC and CC 'will permit comparability between the results of independent security evaluations. It does so by providing a common set of requirements for the security functions of IT products and systems and for assurance measures applied to them during a security evaluation. The evaluation process establishes a level of confidence that the security functions of such products and systems and the assurance measures applied to them meet these requirements.' ([8], Part 1, Scope). A product or system which has been successfully evaluated is awarded with an internationally recognized IT security certificate.

Although random numbers play an important role in numerous cryptographic applications, ITSEC, Common Criteria ([8]) and the corresponding evaluation manuals do not specify any uniform evaluation criteria for random number generators. However, rules are needed for the evaluation of true (physical) random number generators. The AIS 31 (219]) supports this goal. It is mandatory in the German evaluation and certification scheme if the TRNG affects the security properties of the target of evaluation (TOE).

In the following sections we will work out the general objectives formulated above. We will often refer to and cite important parts of [19] where concrete criteria, statistical tests and decision rules are given that a TRNG should fulfil. 


\section{Assessing the Random Number Generation I (Standard Case)}

The minimal requirements the external random numbers should at least fulfil depend essentially on the particular application. In general the application reads the external random numbers from the RNG asynchronous to the generation of the internal random numbers. Therefore a TRNG evaluation can only consider the properties of the das random numbers or the internal numbers. However, as the external random numbers usually are obtained by concatenating internal random numbers this is no serious restriction.

If the external random numbers serve as challenges or as openly transmitted initialization vectors for symmetric block ciphers, for instance, it is fully sufficient if the statistical behaviour of the internal random numbers is similar to that of random numbers generated by an ideal source. Generically, in [19] (P1.c) this reads as follows: 'The statistical behaviour of the internal random numbers should be inconspicious. This shall prevent replay and correlation attacks against cryptographic algorithms and protocols that are based on statistical weaknesses in the external random numbers used.' More concrete, a TRNG is said to be a P1-TRNG in the sense of [2] if its internal random numbers pass a particular statistical test suite (cf. [19], Requirements P1.d(i),(ii) and P1.i(i),(ii)).

Good statistical properties of the internal random numbers are clearly not sufficient for sensitive applications as the generation of session keys, signature key pairs or signature parameters, for example. Note that even output sequences of linear feedback shift registers (LFSR) should pass the P1-specific statistical tests (and many others, cf. Example 1) unless the length $m$ of the LFSR is extremely small. The overall entropy of a pseudorandom number sequence generated by a LFSR is contained in its seed. If a potential attacker knows about $m$ output bits (e.g. from random numbers used as an openly transmitted challenge) he can easily compute all random numbers ever generated. All he has to do is to solve a system of linear equations.

The key criterion is not the statistical behavior of the internal numbers but their entropy. Consequently, in [19] a subclass of the P1-TRNGs is introduced, called P2. For a P2-TRNG evidence has to be given that the increase of entropy per internal random number is sufficiently large. In [19], P2.c, the aim of the P2-specific requirements (cf. [19], P2.d)) is formulated generically: 'In addition to the P1-specific aim P1.c), the prospects of success for systematic guessing of the external random numbers (realised through sytematic exhaustion attacks) - even if external random number subsequences are known - should at best be negligibly higher than would be the case if the external random numbers had been generated by an ideal random number generator.'

To assure this goal the increase of entropy per internal random number must be sufficiently large. Unlike the computational complexity for deterministic random number generators (cf. Sect.2) entropy yields a theoretical security bound. However, entropy is a property of random variables but not of their realizations, in our context, of the observed internal random numbers. Unfortunately, there is no statistical test or reliable estimator (and it is hardly to believe that there 
might exist one) for the case where nothing is known about the distribution of the underlying random variables.

Remark 2. The adjective 'universal' in the title of [23] has led to misunderstanding and confusion in the past. To apply Maurer's test the random numbers are concatenated and interpreted as a bit stream which in turn is segmented into blocks of equal size. If the block size tends to infinity Maurer's test value yields an estimator for the increase of entropy per random bit provided that the bits were generated by a stationary binary random source with finite memory (cf. also [1]). If this assumption is not fulfilled, however, Maurer's test value need not yield a reliable estimator for the entropy. For pseudorandom bits generated by a LFSR, for example, the increase of entropy per bit obviously equals zero whereas the test value 'suggests' a considerable amount of entropy per bit. We point out that 19 indeed applies a test introduced by Coron (cf. 10]) which is closely related with Maurer's test but yields information on the entropy per bit for fixed block size. As will be explained below Coron's test is applied to the das random numbers not until the respective random variables have shown to be (at least approximately) Markovian.

Definition 1. A realization of a random variable $X$ is a value assumed by $X$. $W e$ call a random variable binary if it only assumes the values 0 and 1 . If $X$ assumes values on a finite set $\Omega$ then

$$
H(X):=-\sum_{x \in \Omega} \operatorname{Prob}(\mathrm{X}=\mathrm{x}) \log _{2}(\operatorname{Prob}(\mathrm{X}=\mathrm{x}))
$$

is the entropy of $X$. In the context of random variables iid stands for 'independent and identically distributed'. Applying a statistical test to a sample $x_{1}, x_{2}, \ldots, x_{N}$ delivers a numerical value called test value or test statistic $t$. The test value itself may be interpreted as a realization of a random variable $T$, the so-called test variable.

Mathematical Model. In the following we interpret the das random numbers $b_{1}, b_{2}, \ldots \in \Omega_{\mathrm{das}}$ (usually $\Omega_{\mathrm{das}}=\{0,1\}^{k}$ for $k \geq 1$ ) as realizations of random variables $B_{1}, B_{2}, \ldots$ Similarly, the internal random numbers $r_{1}, r_{2}, \ldots$ are viewed as realizations of random variables $R_{1}, R_{2}, \ldots$ The random variables $R_{1}, R_{2}, \ldots$ result from $B_{1}, B_{2}, \ldots$ by applying the postprocessing algorithm.

Remark 3. The term

$$
\begin{gathered}
H\left(B_{n} \mid B_{1}, \ldots, B_{n-1}\right):=-\sum_{b_{1}, \ldots, b_{n-1} \in \Omega_{\text {das }}} \operatorname{Prob}\left(\mathrm{B}_{1}=\mathrm{b}_{1}, \ldots, \mathrm{B}_{\mathrm{n}-1}=\mathrm{b}_{\mathrm{n}-1}\right) \times \\
\sum_{b_{n} \in \Omega_{\mathrm{das}}} \operatorname{Prob}\left(\mathrm{B}_{\mathrm{n}}=\mathrm{b}_{\mathrm{n}} \mid \mathrm{B}_{\mathrm{j}}=\mathrm{b}_{\mathrm{j}} \text { for } \mathrm{j}<\mathrm{n}\right) \log _{2}\left(\operatorname{Prob}\left(\mathrm{B}_{\mathrm{n}}=\mathrm{b}_{\mathrm{n}} \mid \mathrm{B}_{\mathrm{j}}=\mathrm{b}_{\mathrm{j}} \text { for } \mathrm{j}<\mathrm{n}\right)\right)
\end{gathered}
$$

quantifies the increase of the total entropy of $B_{1}, \ldots, B_{n-1}$ by $B_{n}$. If the random variables $B_{1}, B_{2}, \ldots$ are iid the conditional probabilities do not depend on 
the conditions. If $B_{1}, B_{2}, \ldots$ form a homogeneous Markov chain the conditional probabilities do only depend on the preceding value $b_{n-1}$, and for sufficiently large $n$ we have $\operatorname{Prob}\left(\mathrm{B}_{\mathrm{n}-1}=\mathrm{b}_{\mathrm{n}-1}\right) \approx \nu\left(\mathrm{b}_{\mathrm{n}-1}\right)$ where $\nu$ stands for the stationary distribution (under the natural assumption that the Markov chain is ergodic). If the context is clear, i.e. if we consider the whole sequence, we also use the imprecise formulation 'the entropy of $B_{n}$ '. By abuse of language we will often speak of 'the increase of entropy per das random number' or shortly 'the entropy of a das random number'.

A principle question is whether the das random numbers or the internal random numbers should be considered for testing. The latter seems to be near at hand since the internal random numbers are output. Example 10 (cf. 30], Example 1, or [19], Example E.1), however, underlines that this approach may be misleading.

Example 1. Suppose that the TRNG produces binary das random numbers and let a LFSR of length 63 with primitive feedback polynomial be synchronized with the digitization of the analog noise signal. In each time step the feedback shift register outputs an internal random number (a single bit). The actually generated das random number is XOR-ed to the feedback value, and this sum is fed back into the LFSR. This mathematical postprocessing is a one-to-one mapping for each initial value of the LFSR and hence cannot increase the average entropy per bit. Consequently, weaknesses of the das random numbers cannot be reduced but only transferred into others. If, for example, the das random numbers are independent but not equidistributed (i.e., if the probability for " 0 " is not 0.5 ) the internal random numbers are basically equidistributed but dependent. Unless its linear complexity profile is considered statistical tests applied to the internal random number sequence will presumably not even detect the worst case when the physical noise source has totally broken down. In fact, from this moment on the das random numbers are constant, and the internal random numbers are generated deterministically.

Example1 1underlines an important fact: Even if the internal random numbers pass certain statistical tests which the das random numbers do not this does not necessarily imply that the mathematical postprocessing improves the quality of the das random numbers. Weaknesses may be merely masked and transformed into others. Clearly, an increase of entropy per bit can only be achieved by a data compression which in turn lowers the bit rate.

Of course, also the das random numbers may not be equidistributed and there may exist dependencies on predecessors. However, in contrast to the internal random numbers there will not exist complicated algebraic dependencies. Consequently, the das random numbers should always be tested if this is possible. As demonstrated above internal random numbers may pass statistical tests even if their overall entropy equals zero.

The aim of a P2-evaluation is clear: Evidence shall be given that the increase of entropy per bit is sufficiently large. The crucial requirement a TRNG has to fulfil in the context of entropy is Requirement P2.d)vii): 'Digitised noise signal 
sequences (das random numbers) meet particular criteria or pass statistical tests intended to rule out features such as multi-step dependencies. Moreover, the entropy test T8 is passed. The tests and evaluation rules are specified in subsection P2.i)...'. For the normal case where the TRNG generates a single das bit per time unit in [19] (P2.i)vii)) five tests and an overall decision rule are specified which shall assure this goal. Test $\mathrm{T} 7$ represents a special case of Test 76 (a test for the equality of multinomial distributions) from [17]. In our context (comparison of two bit sequences $a_{1}, \ldots, a_{n}$ and $a_{1}^{\prime}, \ldots, a_{n}^{\prime}$ ) the test value is given by

$$
t_{7}:=\sum_{t=0,1} \frac{\left(h[t]-h^{\prime}[t]\right)^{2}}{h[t]+h^{\prime}[t]}
$$

where $h[t]:=\left|\left\{j \leq n \mid a_{j}=t\right\}\right|$ and $h^{\prime}[t]:=\left|\left\{j \leq n \mid a_{j}^{\prime}=t\right\}\right|$. Under the null hypothesis, i.e. that the bit sequences $a_{1}, \ldots, a_{n}$ and $a_{1}^{\prime}, \ldots, a_{n}^{\prime}$ are realizations of iid binary-valued random variables $A_{1}, \ldots, A_{n}$ and $A_{1}^{\prime}, \ldots, A_{n}^{\prime}$, the test variable $T 7$ is asymptotically $\chi^{2}$-distributed with one degree of freedom. Note that the random variables $A_{1}, \ldots, A_{n}, A_{1}^{\prime}, \ldots, A_{n}^{\prime}$ need not be equidistributed on $\{0,1\}$. Especially, for significance level $\alpha:=0.0001$ the null hypothesis is rejected if $t_{7}>15.13$.

In [19] the following test procedure is specified:

P2.i) (vii.a)The evaluator generates a digitised noise signal (bit) sequence $w_{1}, \ldots$, $w_{n_{0}}$ with $n_{0}:=100000$. Let $\mu_{\mathrm{e} m p}=\left(\mu_{\mathrm{e} m p}(0), \mu_{\mathrm{e} m p}(1)\right)$ be its empirical distribution (i.e. $\left.\mu_{\mathrm{e} m p}(1):=\sum_{j=1}^{n_{0}} w_{j} / n_{1}\right)$. Property (vii.a) is fulfilled if $\left|\mu_{\mathrm{e} m p}(1)-0.5\right|<a_{0}:=0.025$.

P2.i)(vii.b) The evaluator generates a further digitised noise signal sequence $w_{1}, w_{2}, \ldots$ which he splits into 2 disjoint sub-sequences $\mathrm{TF}_{(0)}$ and $\mathrm{TF}_{(1)}$. Here, the tuple $\left(w_{2 j+1}, w_{2 j+2}\right)$ belongs to sub-sequence $\mathrm{TF}_{(r)}$ if and only if $w_{2 j+1}=r$. The initial sequence $w_{1}, w_{2}, \ldots$ must be sufficiently long that both sub-sequences contain at least $n_{1}:=100000$ elements. If we project the first $n_{1}$ 2-tuples of sub-sequence $\mathrm{TF}_{(r)}$ onto the second component, we obtain the one-dimensional sample $\mathrm{St}_{(r)}$. If we divide the frequencies at which individual values ( 0 or 1 ) are assumed by the size of the sample $n_{1}$, we obtain the empirical 1-step transition distribution $\nu_{\mathrm{e} m p(r)}(\cdot)$ for predecessor $r$. Property (vii.b) is fulfilled if $\left|\nu_{\mathrm{e} m p(0)}(1)+\nu_{\mathrm{e} m p(1)}(0)-1\right|<a_{1}:=0.02$.

P2.i)(vii.c) The evaluator generates a further digitised noise signal sequence $w_{1}, w_{2}, \ldots$ which he splits into $2^{2}=4$ disjoint sub-sequences $\mathrm{TF}_{((0)-(0))}, \ldots$, $\mathrm{TF}_{((1)-(1))}$. Here, the triple $\left(w_{3 j+1}, w_{3 j+2}, w_{3 j+3}\right)$ belongs to sub-sequence $\mathrm{TF}_{((r)-(s))}$ if and only if $\left(w_{3 j+1}, w_{3 j+2}\right)=(r, s)$. The initial sequence $w_{1}, w_{2}$, ... must be sufficiently long that each of these four sub-sequences contains at least $n_{2}:=100000$ elements. If we project each of the first $n_{2} 3$-tuples of sub-sequence $\mathrm{TF}_{((r)-(s))}$ onto the third component, we obtain the onedimensional sample $\mathrm{St}_{((r)-(s))}$. For each $s \in\{0,1\}$ the evaluator compares the underlying distributions of the two samples $\mathrm{St}_{((0)-(s))}$ and $\mathrm{St}_{((1)-(s))}$ with test $T 7$ (cf. (11)) at the significance level $a_{2}:=0.0001$ for equality. Property (vii.c) is fulfilled if both tests are passed. Otherwise Property (vii.c) is considered not to be fulfilled. 
P2.i)(vii.d) The evaluator generates a further digitised noise signal sequence $w_{1}, w_{2}, \ldots$ which he splits into 8 disjoint sub-sequences $\mathrm{TF}_{((0)-(0)-(0))}, \ldots$, $\mathrm{TF}_{((1)-(1)-(1))}$. Here, the quadruple $\left(w_{4 j+1}, w_{4 j+2}, w_{4 j+3}, w_{4 j+4}\right)$ belongs to sub-sequence $\mathrm{TF}_{((r)-(s)-(t))}$ if and only if $\left(w_{4 j+1}, w_{4 j+2}, w_{4 j+3}\right)=(r, s, t)$. The initial sequence $w_{1}, w_{2}, \ldots$ must be sufficiently long that each of these eight sub-sequences contains at least $n_{3}:=100000$ elements. If we project each of the first $n_{3}$ quadruples of sub-sequence $\mathrm{TF}_{((r)-(s)-(t))}$ onto the fourth component, we obtain the one-dimensional sample $\mathrm{St}_{((r)-(s)-(t))}$. For each pair $(s, t) \in\{0,1\}^{2}$ the evaluator compares the underlying distributions of the two samples $\mathrm{St}_{((0)-(s)-(t))}$ and $\mathrm{St}_{((1)-(s)-(t))}$ with test $\mathrm{T} 7$ at the significance level $a_{3}:=0.0001$ for equality. Property (vii.d) is fulfilled if all four tests are passed. Otherwise Property (vii.d) is considered not to be fulfilled.

P2.i)(vii.e) The evaluator generates a further digitised noise signal sequence $w_{1}, w_{2}, \ldots$ and applies to it the entropy test (test T8 in [19]; cf. [10]) with the parameters $L=8, Q=2560$ and $K=256000$. Property (vii.e) is fulfilled if the test variable $f>7.976$.

Decision rule: If properties P2.i)(vii.a) - (vii.e) are fulfilled, then Property P2.d)(vii) is considered to be fulfilled. If more than one sub-property is not fulfilled, then Property P2.d)(vii) is considered not to be fulfilled. If precisely one sub-property is not fulfilled, P2.i)(vii.a) - (vii.e) are applied to another sample. If all sub-properties P2.i)(vii.a) - (vii.e) are fulfilled upon repetition, then Property P2.d)(vii) is considered to be fulfilled. A further repetition is not allowed.

We give a brief rationale for the criteria P2.i)(vii.a) to P2.i)(vii.e) and the decision rule. Criterion P2.i)(vii.a) compares the one-dimensional distribution of the das bits with the uniform distribution on $\{0,1\}$ where any dependencies from predecessors are not taken into account. If the sequence $B_{1}, B_{2}, \ldots$ is iid and if $\mu(1):=\operatorname{Prob}\left(\mathrm{B}_{\mathrm{j}}=1\right) \in[0.475,0.525]$ then the entropy per das bit is $>0.998$. The sample size $n_{0}$ was chosen so large that criterion P2.i)(vii.a) is met with a probability of at least $1-0.00078$ if $\mu(1) \in[0.48,0.52]$. Criterion P2.i)(vii.b) considers the one-step transition probabilities. If the random variables $B_{1}, B_{2}, \ldots$ form a homogeneous Markov chain and if the exact transition probabilities $\nu_{(r)}(s):=\operatorname{Prob}\left(\mathrm{B}_{\mathrm{n}}=\mathrm{s} \mid \mathrm{B}_{\mathrm{n}-1}=\mathrm{r}\right)$ meet the inequality $\left|\nu_{(0)}(1)+\nu_{(1)}(0)-1\right|<$ 0.02 the dependencies reduce the entropy at most by 0.00057 per bit (compared with an iid sequence $B_{1}^{\prime}, B_{2}^{\prime}, \ldots$ having the same one-dimensional marginal distribution $\mu$ ). Note that $\nu_{(0)}(1)+\nu_{(1)}(0)=\nu_{(0)}(1)+\nu_{(0)}(0)=1$ if the $B_{j}$ are iid. If $\left|\nu_{(0)}(1)+\nu_{(1)}(0)-1\right|<0.012$ then P2.i)(vii.b) is satisfied with a probability of at least $1-0.00017$.

Remark 4. The das random numbers shall not have deeper than 1-step dependencies from the predecessors. If such dependencies are significant the generation rate of the das bits must be reduced. Slight one-step dependencies are tolerated as those might be caused by dead times of components (e.g. by flip-flops).

Criteria P2.i)(vii.c) and P2.i)(vii.d) shall detect (resp. exclude) possible 2- or 3 -step dependencies. Test T7 compares the conditional distributions $\operatorname{Prob}\left(\mathrm{B}_{\mathrm{n}+2} \mid\right.$ 
$\left.\mathrm{B}_{\mathrm{n}}=0, \mathrm{~B}_{\mathrm{n}+1}=\mathrm{s}\right)$ and $\operatorname{Prob}\left(\mathrm{B}_{\mathrm{n}+2} \mid \mathrm{B}_{\mathrm{n}}=1, \mathrm{~B}_{\mathrm{n}+1}=\mathrm{s}\right)$ and $\operatorname{Prob}\left(\mathrm{B}_{\mathrm{n}+3} \mid \mathrm{B}_{\mathrm{n}}=\right.$ $\left.0, \mathrm{~B}_{\mathrm{n}+1}=\mathrm{s}, \mathrm{B}_{\mathrm{n}+2}=\mathrm{t}\right)$ and $\operatorname{Prob}\left(\mathrm{B}_{\mathrm{n}+3} \mid \mathrm{B}_{\mathrm{n}}=1, \mathrm{~B}_{\mathrm{n}+1}=\mathrm{s}, \mathrm{B}_{\mathrm{n}+2}=\mathrm{t}\right)$, resp., where $r, s \in\{0,1\}$. If the random variables $B_{1}, B_{2}, \ldots$ form a homogeneous Markov chain the respective distributions are equal.

The applicant for an IT certificate (typically the producer of the TRNG) has to 'give clear description how the noise signal is generated, together with an explanation of why a digitised noise signal is to be induced in this way' (19, C.1(iii)). This description shall enable to detect or at least suspect (or, in the positive case, rule out) long-term dependencies of the das random numbers (e.g. caused by a beat). Detecting long-term dependencies by using any blackbox test suite seems to be almost impossible. Similarly, the stationarity assumption shall be made plausible in this way (eventually supported by adapted tests). The Criteria P2.i)(vii.b) to P2.i)(vii.d) shall detect possible short-term dependencies from predecessors. If these tests are passed this is viewed as an evidence that the sequence $B_{1}, B_{2}, \ldots$ is (at least approximately) a stationary Markov chain. (The stationarity assumption shall follow from the explanations mentioned at the beginning of this paragraph.) In particular, this induces the entropy bounds from above for the das random numbers, and the assumptions of Coron's entropy test are fulfilled. To be precise, if $B_{1}, B_{2}, \ldots$ were iid the expectation of Coron's test value equals the entropy per $L$-bit das block.

As the evaluation result is based on statistical tests it cannot be reproduced with certainty. However, it is 'quasireproducible' in the sense that for 'reasonable' TRNGs it is extremely unlikely that Requirement P2.d)vii) is considered not to be fulfilled. The probability for an ideal random number generator not to meet the particular criteria is $0,0,2 \cdot 10^{-4}, 2 \cdot 10^{-4}$, and 0 , resp. The probability that an ideal random number generator is not being attributed Property P2.d)(vii) is about $6 \cdot 10^{-7}$ (cf. P2.i)(vii.e)).

Remark 5. Clearly, the mathematical postprocessing must not reduce the average entropy per bit (Property P2.d)(viii)). (The adjective 'average' is due to the fact that the particular bits of the internal random numbers need not be identically distributed.)

Up to now we have exclusively considered the case that the das random numbers are binary-valued. However, there are TRNGs which generate $k$-bit das random numbers for $k>1$. Unlike for the case $k=1$ Reference [19] does not provide a concrete test suite for $k>1$. However, [19] does not exclude those TRNGs. The applicant rather has to specify appropriate alternative tests which shall not be weaker than those for $k=1$, i.e. they must ensure at least the same entropy bounds per bit. Depending on the noise source and the precise form of digitisation the individual bits of the das random numbers need not be identically distributed and there might exist dependencies between particular bits. A stationary $\{0,1\}^{k}$-valued sequence need not necessarily induce a stationary binary valued sequence when the $k$-bit-values are interpreted as binary subsequences of length $k$. As a consequence, it is not sufficient just to apply the tests from P2.i)(vii.a) to P2.i(vii.e) to the derived binary sequence (interpreting the $k$-bit values as binary subsequences of length $k$ ) without further justifications. 


\section{Assessing the Random Number Generation II (Alternative Criteria)}

In the previous section we claimed and justified that for P2 evaluations the das random numbers should be investigated but not the internal random numbers. The aim of Criteria P2.i)(vii.a)-(vii.e) (or the TRNG-individual criteria if $k>1$, resp.; cf. the final paragraph of the preceding section) was to verify with negligible error probability that the average entropy per das bit exceeds an acceptable minimum limit. Further, the mathematical postprossing shall not reduce the entropy per bit (cf. Remark 5). Note that there exist TRNGs for which the das random numbers do not meet these requirements (Case 1), or due to the construction of the TRNG the evaluator may not have access to the das random numbers (Case 2). However, TRNGs of this kind need not necessarily be inappropriate. For Case 1, for instance, a suitable postprocessing might increase the entropy per bit. The AIS 31 does not automatically exclude such TRNGs. It is nonetheless conceded that a TRNG meets Requirement P2.d)(vii) (cf. Sect. 4) if the applicant is able to specify and justify suitable alternative evaluation criteria.

In Case 1 the tests destined for the das random numbers have to be applied to the internal random numbers. Additionally, and this is the crucial point which may turn out to be difficult or even impossible for a concrete TRNG, in [19] (Alternative criteria for P2.d)(vii); type 1)) it is specified that the applicant has to give 'Clear Proof that the internal random numbers achieve the goal set with criterion P2.d)(vii). The proof must be provided taking into account the mathematical postprocessing and on the basis of the empirical properties of the digitised noise sequence'.

The 'clear proof' can be based on statistical tests of the internal random numbers if their suitability is justified. Depending on the type of postprocessing this may be rather difficult or even impossible (cf. Example 2 below). In Case 2 the situation is even more difficult. Again, the tests destined for the das random numbers have to be applied to the internal random numbers. Additionally, in [19] (Alternative criteria for P2.d)(vii); type 2)) it is specified that the applicant has to give 'Comprehensible and plausible description of a mathematical model of the physical noise source and the statistical properties of the digitised noise signal sequence derived from it.' Further, he has to give a 'Specification of statistical tests that guarantee the goal defined in criterion P2.d)(vii) insofar as the internal random numbers pass these tests. It shall be comprehensibly justified that these tests are suitable. The proof must be provided taking into account the mathematical postprocessing and on the basis of the statistical properties of the noise signal sequence derived from the mathematical model of the noise source.'

Reference [19] explicitly permits alternative criteria since no reasonable random number generator should be excluded from getting a certificate. It is not the intention of [19] to favour or discriminate particular types of TRNGs. The applicant himself has to specify and justify alternative criteria since it should be reasonable to expect that he is able to give evidence why his TRNG design 
is suitable for sensitive cryptographic applications. If the evaluator has access to the das random numbers (single bits) and if the das random numbers fulfil the Criteria P2.i)(vii.a)-(vii.e) his work has already been done in [19] (cf. Sect. 4). The following example (cf. [19], Example E.4) illustrates the procedure and general difficulties when alternative criteria are applied.

Example 2. Throughout this example we assume that the noise source generates binary-valued das random numbers. We consider three different mathematical postprocessings.

a) The das random numbers (bits) are XORed to the feedback value of an LFSR (cf. Example1).

b) Non-overlapping pairs of consecutive das bits are XORed.

c) The das bit sequence $b_{1}, b_{2}, \ldots$ is segmented into non-overlapping blocks $y_{1}, y_{2}, \ldots$ of length 128 . The internal random numbers are given by $r_{j}:=$ $\operatorname{AES}\left(y_{2 j-1}, y_{2 j}\right)$, i.e. block $y_{2 j-1}$ is encrypted with key $y_{2 j}$.

(i) (Case 1) Suppose that extensive investigations of TRNG prototypes have shown that the das bits may be viewed as realizations of iid random variables but the (prototype-dependent) probability for assuming the value "1" lies in the interval $[0.45,0.47]$. Obviously, the das random numbers do not meet criterion P2.i(vii.a).

Postprocessing a) does not increase the entropy per bit and hence an evaluation with alternative criteria is definitely not possible. Now assume that variant b) is applied. As there is evidence that the das random numbers are independent the internal random numbers should also behave like realizations of independent random variables, and the probability for a " $1 "$ lies in the interval [0.49875, 0.50125]. This argumentation provides a comprehensible proof sufficient for a Case 1 evaluation with alternative criteria. Consequently, the TRNG is conceded to meet Property P2.d)(vii). For variant c) statistical tests of the internal random numbers cannot deliver any useful information because such tests had at least to take the one-dimensional distribution of 128-bit blocks into consideration. An evaluation with alternative criteria would instead require a theoretical proof that the mathematical postprocessing leads to a sufficient increase of entropy per bit.

(ii) (Case 2) Suppose that a careful analysis of the precise realization of the physical noise source (taking into account the switching times and dead times of individual building blocks, sampling rates etc.) made it plausible to assume that the das random numbers are independent. (A comprehensible justification for deriving this mathematical model is extremely important!)

Postprocessing a) can easily be back-calculated, and Criteria P2i).(vii.a)-(vii.e) can be applied to the das random numbers. As the postprocessing does not increase the entropy per bit a Case 2 evaluation is only possible if the das bits fulfil these criteria. Similarly as above we can argue that variant b) transforms the das bits into independent internal random numbers. Consequently, it is necessary and sufficient that the internal random numbers fulfil the Criteria P2.i)(vii.a)(vii.e). For c) the situation is even more complicated than in (i). An evaluation with alternative criteria seems hardly be possible. 


\section{$6 \quad$ Startup Test, Online Test, Tot Tests}

In the previous sections we have intensively considered criteria for assessing the suitability of the random number generation process. However, even if Property P2.d)(vii) is conceded, i.e. if the prototypes in the lab generated 'high-quality' random numbers this may not be true for any TRNG of the same production run all the time when it is in operation. Tolerances of components of the noise source or aging effects may be responsible for this. In the worse case the noise source breaks totally down so that the das random numbers are constant from that moment on. Therefore, the developer should implement measures which shall detect defects of this kind in case they occur. We distinguish between tot tests ('tot' stands for 'total failure of the noise source'), startup tests, and online tests. As their names indicate a tot test shall detect a total breakdown of the noise source, the startup test is used to verify the principle functionality of the noise source when the TRNG has been started whereas the online test should detect if the quality of the random numbers is not sufficient for this particular TRNG (due to tolerances of components) or deteriorates in the course of the time.

Remark 6 . The properties of the random number generation determines the class (P1,P2) to which the TRNG belongs. Besides, ITSEC and CC consider the 'strength of mechanisms and functions' as resistance against direct attacks. It is distinguished between 'low', 'medium' and 'high' and is assessed by means of the attack potential which is a function of expertise, resources and motivation required for a success ([9], Annex B.8). In our context the conceded strength of mechanisms or functions depends on the suitability to resist direct attacks exploiting weaknesses in the implementation. The tot, startup and online test are countermeasures to prevent negative effects of errors or failure of the TRNG or of malicious external influences on the quality of the generated random numbers (cf. Sect. 7). In such cases the random number generation should be stopped.

If the strength of mechanism (cf. [15]) or functions (cf. 8] ) is low [19] does not demand any tot test, startup test or online test. If the strength is medium or high these tests are required. For class P1 these tests shall consider the internal random numbers (cf. [19], P1.d)). We will not pursue this aspect in the following. Instead, we will concentrate on class P2 with strength of mechanisms high.

The requirement on the startup test are rather mild. It only has to verify statistical minimum properties of the das random numbers when the TRNG has been started (19], P2.d)(ix)). The tot test has to prohibit that random number sequences are output for which the underlying das random numbers have been generated completely after a total failure of the noise source ([19], P2.d)(x); cf. also Remark 7(i)). The online test has to check the quality of the das random numbers. The TRNG has to trigger the online test itself. The online test and the call schema must be suitable for detecting unacceptable statistical defects of the das random numbers or the deterioration of their statistical properties within an acceptable period of time. For ideal random number generators the probability 
that at least one random alarm occurs in the course of a year of typical use of the particular TRNG should be $\geq 10^{-6}$ ([19], P2.d)(xi),(xiii)).

Remark 7. (i) Ideally, no external random number should be output if any of the used das random numbers has been generated after a total breakdown of the noise source. This aim can be assured by using only internal random numbers where an online test (applied to the das random numbers) has been passed after these internal numbers had been generated. The internal random numbers should be generated from the das random numbers of the beginning of the test sample. However, this is not always possible. The minimal requirement P2.d)(x) in [19] needs concrete analysis for specific technical solutions.

(ii) If the internal random numbers are tested instead of the das random numbers an extra justification for the suitability of the tests is necessary (cf. [19], Comment 2).

(iii) A minimum probability for an erroneous noise alarm was specified in order to rule out weak statistical tests; e.g. if a noise alarm was released iff 64 consecutive das random numbers are equal. (However, this might be an appropriate decision rule for the tot test.)

(iv) The tot test, startup test and the online test are usually a part of the TRNG implementation. In exceptional cases they may be realized as external security measures, e.g. by software calling the TRNG. In this case the applicant has to provide an exact specification of these measures and a reference implementation ([19], Comments 1 and 3).

(v) Due to the small alarm probabilities the effectiveness of the online test has to be justified on a theoretical basis.

The AIS 31 does not prescribe any concrete solutions. Reference [19] merely demands properties tot tests, startup tests and online tests should have. Moreover, various examples are discussed ([19], Examples E.5-E.7).

Remark 8. (i) For the sake of efficiency the tot test, startup test and online test may use the same statistical test, although with different decision rules (cf. [30. or [19], Example E.7).

(ii) Many TRNGs release a noise alarm if a single test value exceeds a certain limit which is expected to occur with an extremely small probability (e.g., $\leq 10^{-8}$ ). As pointed out in [30. (Sect. 4) this approach has two drawbacks: Even for ideal random number generators usually only an approximation of the distribution of the test variable is known (e.g. for the $\chi^{2}$-test). At the tail of these distributions the relative error may be very large. Moreover, those approximations normally give only little information on the rejection probability if the random numbers are not independent and equidistributed.

(iii) In 30] (cf. also 19], Example E.7) a new online test procedure was proposed where it is practically feasible to determine the expected number of noise alarms within a time interval, even if the tested random numbers are not independent and equidistributed. Moreover, the system designer can vary a whole parameter set and hence can fit the test to the very special requirements of the intended applications. Compared with the widely used online tests mentioned in (ii) the 
proposed solution does only need little more memory, some additional lines of code and slightly more running time. These aspects are of particular importance if the TRNG is integrated in a smart card.

\section{Vulnerability Analysis}

The evaluation process according to CC (cf. 8] and [9]) or ITSEC (cf. [15] and [16]) will provide evidence for the correct implementation and the understanding of the design. The statistical tests and the criteria described in Sects. 4 and 5 check the properties of the random numbers generated by prototypes. The effectiveness of the tot, startup and online test is verified mostly by theoretical arguments. The goal of the vulnerability analysis is to determine whether vulnerabilities have been identified during the evaluation of the construction and anticipated operations of the TOE, or whether other methods (e.g. flaw hypotheses) could allow users to violate the security policy of the target of evaluation. Vulnerabilities of the RNG may allow the ability to interfere with or alter the random numbers. The analysis starts with obvious vulnerabilities which are open to exploitation and requires a minimum of understanding of the TOE, skill, technical sophistication, and resources in the public domain.

Note that the evaluation guidelines 19 themselves directly address countermeasures against some obvious vulnerabilities of the TRNG, e.g. aging effects and the total failure of the noise sources by requirements for tot, startup and online tests. Obvious vulnerabilities might be suggested by the RNG external and internal interface description. One easily sees the vulnerability of an hybrid RNG which uses external random data which are under the control of an attacker. Noise sources based on inherent semiconductor thermal noise may be vulnerable to environmental conditions like temperature. The attacker may try to run the RNG at very low or very high temperature which could affect the quality of the random numbers. Requirement [19], P2.d)(xii) demands that Requirement P2.d)(vii) (cf. Sects.4 and 5) are fulfilled under the intended external usage conditions (temperature, power supply etc.) insofar as these can influence the function of the noise source. If the environmental conditions are hostile, it may be necessary to extend the tests under the aspect of the strength of mechanisms and the analysis of the weaknesses. Smart card chips are normally protected by means of a temperature sensor against operation out of range. The evaluator should examine whether this range of temperature is valid for the normal operation of the RNG. In some cases the online tests may detect deviations from normal RNG operations more effective than the sensor, especially if aging of the random source is taken into account. The obvious vulnerability get more specific if the concrete product is analyzed.

Another example of potential vulnerabilities of a TRNG (at least if it is implemented on a smart card chip) are side channels caused by the physical random source or the postprocessing. A vulnerability analysis of pseudo-random number generators may be found in [18]. A very specific but very instructive example of a DRNG vulnerability is described in [5]. The authors show that if 
the random parameter $k$ in the Digital Signature Algorithm (DSA) is generated by means of a linear congruential DRNG then the private key can quickly be recovered from a few signatures even though this generator passes all statistical tests and none of the pseudo-random numbers is ever revealed. D. Bleichenbacher [6] discovered a flaw in the RNG described in the Appendix 3 of FIPS 186-2 that result in the non-uniformity of the generated pseudo-random numbers. The RNG is revised in the change notice to FIPS 186-2 ([26]).

The vulnerability analysis has to consider all information obtained during the evaluation process of the TOE so far. All vulnerabilities have to be assessed whether they might be used for practical attacks. One aspect is the expected number of trials which are necessary to guess random numbers. The optimal strategy in an exhaustive key search begins with the most likely bit string and continues guessing in order of decreasing probabilities. Let $Z$ denote a random variable assuming values in $\left\{z_{1}, z_{2}, \ldots, z_{2^{n}}\right\}$, the set of $n$-bit strings, ordered with respect to their probabilities, i.e. $P\left(z_{1}\right) \geq P\left(z_{2}\right) \geq \cdots P\left(z_{2^{n}}\right)$. For $0 \leq \delta \leq 1$ let $\mu(n, \delta)$ denote the minimum number of strings which must be guessed in order to find the correct string with a probability of at least $\delta$. Then clearly

$$
\mu(n, \delta):=\min \left(k \mid \sum_{i=1}^{k} P\left(z_{i}\right) \geq \delta\right) .
$$

Assume that an RNG may be viewed as an ergodic stationary binary-valued source with $p$ denoting the probability for the value 1 . In [23] it is pointed out that then

$$
\lim _{n \rightarrow \infty} \frac{\log _{2} \mu(n, \delta)}{n}=H(p) .
$$

Generally the RNG need not generate binary-valued random numbers. In the most extreme case it generates each $n$-bit string at one go. Then the strong assumption of the Maurer's formula does not hold. More generally, Pliam's formula (28]) is valid:

$$
\left\lfloor\frac{1}{2 \max _{1 \leq i \leq 2^{n}} P\left(z_{i}\right)}\right\rfloor \leq \mu(n, 0.5) \leq\left\lceil 2^{n}\left(1-\sum_{i=1}^{2^{n}}\left|P\left(z_{i}\right)-2^{-n}\right|\right)\right\rceil .
$$

Depending on the concrete numerical values the evaluator may use the lower bound to show that the RNG is secure against a guessing attack and the upper bound to point at a weakness.

\section{Conclusions}

A comprehensive introduction in the evaluation of physical random number generators was given. Fundamental differences to deterministic random number generators and hybrid number generators were pointed out and general principles for the evaluation of TRNGs have been explained. Concrete measures and criteria specified in [19] were cited and explained. 
The AIS 31 (cf. [219]) has been effective in the German evaluation and certification scheme since September 2001. It is mandatory if a physical random number generator influences the security properties of an IT product and if a German IT security certificate is applied for. The AIS 31 does not favour or exclude particular TRNG design principles. It prescribes properties and criteria a TRNG should fulfil and the evaluation task to be performed.

\section{References}

1. AIS 20: Functionality Classes and Evaluation Methodology for Deterministic Random Number Generators. Version 1 (02.12.1999) (mandatory if a German IT security certificate is applied for; English translation). www.bsi.bund.de/zertifiz/zert/interpr/ais20e.pdf

2. AIS 31: Functionality Classes and Evaluation Methodology for Physical Random Number Generators. Version 1 (25.09.2001) (mandatory if a German IT security certificate is applied for; English translation). www.bsi.bund.de/zertifiz/zert/interpr/ais31e.pdf

3. ANSI X9.17-1985, Financial Institution Key Management (Wholesale).

4. V. Bagini and M. Bucci: A Design of Reliable True Number Generators for Cryptographic Applications. In: Ç.K. Koç and C. Paar (eds.): Cryptographic Hardware and Embedded Systems - CHES 1999. Springer, Lecture Notes in Computer Science, Vol. 1717, Berlin (1999), 204-218.

5. M. Bellare, S. Goldwasser, D. Micciancio: "Pseudo-Random" Number Generation Within Cryptographic Algorithms: The DSS Case. In: B. Kaliski (ed.): Crypto'97. Springer, Lecture Notes in Computer Science, Vol. 1294, Berlin (1997), 277-291.

6. Lucent Technologies, Bell Labs: Scientist discovers significant flaw that would have threatened the integrity of on-line transactions, press article at www.lucent.com/press/0201/010205.bla.html.

7. M. Blum and S. Micali: How to Generate Cryptographically Strong Sequences of Pseudo-Random Bits. SIAM J. Comput., Vol. 13 (1984), 850-864.

8. Common Criteria for Information Technology Security Evaluation, Part 1-3; Version 2.1, August 1999 and ISO 15408:1999.

9. Common Methodology for Information Technology Security Evaluation CEM99/045, Part 2: Evaluation Methodology, Version 1.0 (August 1999).

10. J.-S. Coron: On the Security of Random Sources. In: H. Imai and Y. Zheng (eds.): Public Key Cryptography - PKC 99. Springer, Lecture Notes in Computer Science, Vol. 1560, Berlin (1999), 29-42.

11. J.-C. Coron, D. Naccache: An Accurate Evaluation of Maurer's Universal Test. In: S. Tavares and H. Meijer (eds.): Selected Areas in Cryptography — SAC '98. Springer, Lecture Notes in Computer Science, Vol. 1556, Berlin (1999), 57-71.

12. L. Devroye: Non-Uniform Random Variate Generation. Springer, New York (1986).

13. IEEE: IEEE P1363 / D8 (Draft Version 8), Annex D, Standard Specifications for Public Key Cryptography (05.10.1998).

www.informatik.tu-darmstadt.de/TI/Veroeffentlichung/Artikel/Kryptographie/ PublicKeyAllgemein/P1363-D-10-05-98.pdf

14. Intel Platform Security Division: The Intel Random Number Generator. Intel Corporation (1999).

15. Information Technology Security Evaluation Criteria (ITSEC); Provisional Harmonised Criteria, Version 1.2, June 1991 
16. Information Technology Security Evaluation Manual (ITSEM); Provisional Harmonised Methodology, Version 1.0, September 1993

17. G.K. Kanji: 100 Statistical Tests. Sage Publications, London (1995).

18. J. Kelsey, B. Schneier, D. Wagner, C. Hall: Cryptanalytic Attacks on Pseudorandom Number Generators. In: S. Vaudenay (ed.): Fast Software Encryption FSE 1998, Springer, Lecture Notes in Computer Science, Vol. 1372, Berlin (1998), 168-188.

19. W. Killmann, W. Schindler: A Proposal for: Functionality Classes and Evaluation Methodology for True (Physical) Random Number Generators. Version 3.1 (25.09.2001), mathematical-technical reference of [2] (English translation); www.bsi.bund.de/zertifiz/zert/interpr/trngk31e.pdf

20. D.E. Knuth: The Art of Computer Programming. Vol. 2, Addison-Wesley, London (1981).

21. D.P. Maher, R.J. Rance: Random Number Generators Founded on Signal and Information Theory. In: Ç.K. Koç, C. Paar (eds.): Cryptographic Hardware and Embedded Systems - CHES 1999. Springer, Lecture Notes in Computer Science, Vol. 1717, Berlin (1999), 219-230.

22. G. Marsaglia: Diehard (Test Suite for Random Number Generators). www.stat.fsu.edu/ ${ }^{\text {geo/diehard.html }}$

23. U. Maurer: A Universal Statistical Test for Random Bit Generators. J. Crypt. 5 (1992), 89-105.

24. NIST: Security Requirements for Cryptographic Modules. FIPS PUB 140-1 (11.04.1994). www.itl.nist.gov/fipspubs/fip140-1.htm

25. NIST: Security Requirements for Cryptographic Modules. FIPS PUB 140-2 (25.05.2001) and Change Notice 1 (10.10.2001). csrc.nist.gov/publications/fips/fips140-2/fips1402.pdf

26. NIST: Digital Signature Standard (DSS). FIPS PUB 186-2 (27.01.2000) with Change Notice 1 (5.10.2001). csrc.nist.gov/publications/fips/fips186-2/fips186-2-change1.pdf

27. A. Rukhin et al.: A Statistical Test Suite for Random and Pseudorandom Number Generators for Cryptographic Applications. NIST Special Publication 800-22 with revisions dated 15.05.2001. csrc.nist.gov/rng/SP800-22b.pdf

28. J.O. Pliam: The Disparity Between the Work and the Entropy in Cryptology (01.02.1999). eprint.iacr.org/complete/

29. W. Schindler: Functionality Classes and Evaluation Methodology for Deterministic Random Number Generators. Version 2.0 (02.12.1999), mathematical-technical reference of [1] (English translation); www.bsi.bund.de/zertifiz/zert/interpr/ais20e.pdf

30. W. Schindler: Efficient Online Tests for True Random Number Generators. In: Ç.K. Koç, D. Naccache, C. Paar (eds.): Cryptographic Hardware and Embedded Systems - CHES 2001. Springer, Lecture Notes in Computer Science, Vol. 2162, Berlin (2001), 103-117. 